\title{
Microbial infections in eight genomic subtypes of chronic fatigue syndrome/myalgic encephalomyelitis
}

\author{
Lihan Zhang, ${ }^{1}$ John Gough, ${ }^{1}$ David Christmas, ${ }^{2}$ Derek L Mattey, ${ }^{3}$ Selwyn CM Richards, \\ Janice Main, ${ }^{5}$ Derek Enlander, ${ }^{6}$ David Honeybourne, ${ }^{7}$ Jon G Ayres, ${ }^{8}$ David J Nutt, ${ }^{2}$ \\ Jonathan $\mathrm{R}$ Kerr ${ }^{1}$
}

${ }^{1}$ Department of Cellular $\&$ Molecular Medicine, St George's University of London, London, UK

${ }^{2}$ Psychopharmacology Unit, Department of Community Based Medicine, University of Bristol, Bristol, UK ${ }^{3}$ Staffordshire Rheumatology Centre, Stoke on Trent, UK ${ }^{4}$ Dorset CFS Service, Poole Hospital, Dorset, UK ${ }^{5}$ Department of Infectious Diseases and General Medicine, Imperial College London, St Mary's Hospital, London, UK ${ }^{6}$ New York ME/CFS Service, New York, USA

${ }^{7}$ Department of Respiratory Medicine, Birmingham Heartlands Hospital, Birmingham, UK

${ }^{8}$ Department of Environmental and Occupational Medicine, University of Birmingham, Birmingham, UK

\section{Correspondence to} Dr Jonathan R Kerr, Room 2.267, Jenner Wing, St George's University of London, Cranmer Terrace, London SW17 ORE, UK; jkerr@sgul.ac.uk

This work was presented at the International Association for Chronic Fatigue

Syndrome/Myalgic

Encephalomyelitis (IACFSME), Reno, Nevada, USA, March 2009

Accepted 3 November 2009 Published Online First 2 December 2009

\section{UNLOCKID}

This paper is freely available online under the BMJ Journals unlocked scheme, see http://jcp. bmi.com/site/about/unlocked. xhtml.

\begin{abstract}
Background The authors have previously reported genomic subtypes of chronic fatigue syndrome/myalgic encephalomyelitis (CFS/ME) based on expression of 88 human genes.
\end{abstract}

Aim To attempt to reproduce these findings, determine the specificity of this signature to CFS/ME, and test for associations between CFS/ME subtype and infection. Methods Expression levels of 88 human genes were determined in blood of 62 new patients with idiopathic CFS/ME (according to Fukuda criteria), six patients with Q-fever-associated CFS/ME from the Birmingham 0-fever outbreak (according to Fukuda criteria), 14 patients with endogenous depression (according to DSM-IV criteria) and 29 normal blood donors.

Results In patients with CFS/ME, differential expression was confirmed for all 88 genes. 0-CFS/ME had similar patterns of gene expression to idiopathic CFS/ME. Gene expression in patients with endogenous depression was similar to that in the normal controls, except for upregulation of five genes (APP, CREBBP, GNAS, PDCD2 and $P D C D 6)$. Clustering of combined gene data in CFS/ME patients for this and the authors' previous study (117 CFS/ME patients) revealed genomic subtypes with distinct differences in SF36 scores, clinical phenotypes, severity and geographical distribution. Antibody testing for Epstein-Barr virus, enterovirus, Coxiella burnetii and parvovirus B19 revealed evidence of subtype-specific relationships for Epstein-Barr virus and enterovirus, the two most common infectious triggers of CFS/ME.

Conclusions This study confirms the involvement of these genes in CFS/ME.

\section{INTRODUCTION}

Chronic fatigue syndrome/myalgic encephalomyelitis (CFS/ME) is a disease characterised by severe and debilitating fatigue, sleep abnormalities, impaired memory and concentration, and musculoskeletal pain. ${ }^{1}$ In the Western world, the population prevalence is estimated to be of the order of $0.5 \%{ }^{2} 3$ Research studies have identified various features relevant to the pathogenesis of CFS/ME such as viral infection, immune abnormalities and immune activation, exposure to toxins, chemicals and pesticides, stress, hypotension, lymphocyte abnormalities and neuroendocrine dysfunction. However, the precise underlying disease mechanisms and means by which these abnormalities inter-relate in patients with CFS/ME remain to be clarified. ${ }^{45}$

Various groups have analysed the gene expression in peripheral blood of patients with CFS/ME, and, in all of these studies, genes of immunity and defence are prominent. Following a pilot microarray study which identified 16 abnormally expressed genes in CFS/ME, ${ }^{6}$ we reported on a comprehensive microarray study which reveals abnormal expression of 88 human genes in patients with CFS/ME. ${ }^{7}$ Clustering of these data revealed seven genomic subtypes of CFS/ME with distinct differences in SF36 scores, clinical phenotypes, severity and geographical distribution. ${ }^{7} 8$ However, remaining questions relate to reproducibility and the specificity of these gene abnormalities to CFS/ME and possible associations with infectious agents.

In this study, we set out to determine whether these findings were reproducible in fresh subjects, whether the previously reported dysregulation of these genes also occurred in drug-free patients with endogenous depression, and whether there was any relationship between particular microbial infections and CFS/ME genomic subtype. The results show that these findings are reproducible and that gene expression in patients with endogenous depression was markedly different from that in patients with CFS/ME, and was similar to that in the normal controls, in terms of these 88 human genes. Also, clustering of gene data revealed eight genomic subtypes with distinct clinical differences, and several of these had interesting associations with particular microbial infections.

\section{METHODS}

Subject enrolment, clinical characterisation and blood sampling

Patients with CFS/ME ( $\mathrm{n}=62)$, who lived in Birmingham $(n=6)$, Bristol $(n=3)$, London $(n=9)$ and New York $(n=44)$, were diagnosed according to Fukuda diagnostic criteria for CFS/ME ${ }^{1}$ and enrolled into the study. All had idiopathic CFS/ME except the six Birmingham patients, who had CFS/ME that had been triggered by laboratory-documented Q fever. Patients with psychiatric disease were excluded using the Minnesota International Neuropsychiatric Interview, thus ensuring that none of our CFS/ME patients had major psychiatric disease or misused alcohol or other drugs. Clinical and quantitative PCR (qPCR) data for these new patients were combined with those for $55 \mathrm{CFS} / \mathrm{ME}$ patients from a previous study, ${ }^{7}$ giving a total of 117 CFS/ME patients, who lived in Birmingham $(n=6)$, Bristol $(n=14)$, Leicester $(n=1)$, London $(n=12)$, New York $(n=55)$ and Dorset $(n=28)$.

Patients with endogenous depression $(n=14)$ were enrolled from Bristol, UK, and surrounding area. These patients fulfilled Diagnostic and statistical manual of mental disorders, 4th edition (DSM-IV) criteria, had not 
smoked within the previous year, and had not taken antidepressants in the previous year.

Healthy normal blood donors enrolled from the Dorset National Blood Service $(n=29)$ were used as a comparison group. Restrictions imposed by the Dorset National Blood Service on those allowed to donate blood are outlined elsewhere. ${ }^{6}$

For all patient groups, individuals who smoked in the previous year, who abused alcohol or other drugs, or were currently taking (or were within 3 months of taking) antibiotics, steroids, cytotoxic drugs or antidepressants were excluded from the study.

For all enrolled subjects (patients and controls), according to the recommendations of the International CFS Study Group, ${ }^{9}$ severity of physical and mental fatigue was assessed using the Chalder Fatigue Scale, ${ }^{10}$ level of disability was assessed using the Medical Outcomes Survey Short Form-36 (SF36), accompanying symptoms were characterised using the Somatic and Psychological Health Report, sleep abnormalities were assessed using the Pittsburgh Sleep Questionnaire, and assessment of type and severity of pain was performed using the McGill Pain Questionnaire.

Patients and controls gave informed written consent according to guidance of the Wandsworth Research Ethics Committee (approval number 05/Q0803/137). For the New York patients, approval of the local institutional review board was obtained. The human experimentation guidelines of the US Department of Health and Human Services were followed in this study.

A $2.5 \mathrm{ml}$ sample of blood was taken from both CFS/ME patients and normal blood donors (as part of routine blood donation) into PAXgene tubes (PreAnalytix, Qiagen, UK), and total RNA extracted using the PAXgene blood RNA kit (PreAnalytix), according to the instructions of the manufacturer. RNA quality and amount were confirmed by micro-spectrophotometry (Nanodrop, Rockland, Delaware, USA). Total RNA samples used in this study had an absorbance ratio $\left(\mathrm{A}_{260} / \mathrm{A}_{280}\right)$ of $1.9-2.0$.

\section{Quantitative PCR}

qPCR (Applied Biosystems, Foster City, California, USA) was used to quantitate the amount of mRNA for 88 CFS/ME-associated human genes by the comparative method, using custom 384-well low-density arrays and the ABI PRISM 7900HT instrument (Applied Biosystems), with glyceraldehyde-3-phosphate dehydrogenase as the endogenous control gene. Experiments were performed in triplicate using the protocol described previously. ${ }^{6}$ Data were displayed using SDS v2.2 software (ABI), discordant data between replicates were omitted, and results for each low-density array were calculated and loaded into ABI SDS v2.2 Enterprise Edition software.

The threshold cycle $(\mathrm{Ct})$ for each test gene in each sample was compared with that for glyceraldehyde-3-phosphate dehydrogenase to calculate a $\Delta \mathrm{Ct}$ value. $\Delta \mathrm{Ct}$ values were then normalised to the calibrator sample to give the $\Delta \Delta \mathrm{Ct}$ values. Relative quantities (RQ) $\left(2^{-\Delta \Delta \mathrm{Ct}}\right)$ of each mRNA of interest were then calculated. Samples showing a difference between minimum and maximum $R Q$ values of $\geq 100$ (indicating poor replicate concordance) were excluded. The t test was used to compare mean RQ values between groups. $p \leq 0.05$ was taken to be significant.

\section{Clustering of qPCR-generated gene values of CFS/ME patients}

$\mathrm{Ct}$ values for all $88 \mathrm{CFS} / \mathrm{ME}$-associated genes in $117 \mathrm{CFS} / \mathrm{ME}$ patients were then normalised and clustered using Genesis software. ${ }^{11}$ For each of the eight CFS/ME subtypes identified using this approach, mean $\mathrm{RO}$ values were calculated for each gene, and used to generate fold-difference (CFS/ME/normal) values for each gene in each CFS/ME subtype. Mean fold-difference values for each gene in each CFS/ME subtype were then clustered with and without normalisation/median centring using Cluster v2.11 software and visualised using Treeview v1.60 software. ${ }^{12}$ The clustering algorithm in both of these software programs has been described previously. ${ }^{12}$

\section{Detection of anti-microbial antibodies}

IgM and IgG antibodies specific to four microbes that are well recognised to trigger CFS/ME were detected by ELISA, according to the manufacturer's instructions: Epstein-Barr virus (EBV) (viral capsid antigen (VCA) IgM and IgG, early antigen IgG and Epstein-Barr nuclear antigen (EBNA) IgG; Meridien Bioscience Inc, Cincinnati, Ohio, USA), enterovirus (all serotypes; Virion Serion, Wurzberg, Germany), parvovirus B19 (viral protein 2 IgM and IgG; Biotrin, Dublin, Ireland) and Coxiella burnetii (phase I and II IgG; Virion Serion).

\section{Statistical testing}

Testing of the significance of associations of gene expression levels with different patient groups was performed using a twotailed $t$ test. Testing of the significance of association between clinical variables and CFS/ME genomic subtype was performed using $\chi^{2}$, analysis of variance (ANOVA) and the Mann-Whitney $U$ tests. Testing of the significance of association between microbial markers in CFS/ME and CFS/ME subtypes was performed using $\chi^{2}$ analysis and ANOVA.

\section{RESULTS}

\section{Subjects and clinical characterisation}

A total of 117 patients with CFS/ME fulfilling Centers for Disease Control diagnostic criteria were used in this study. For 55 , previously published data were used, while the remaining 62 had not previously been tested; for six of these, CFS/ME disease had been triggered by laboratory-documented $C$ burnetii infection. In addition, 14 patients with endogenous depression and 29 normal blood donors were studied.

A summary of the clinical details of these subjects is shown in table 1. In general, all CFS/ME groups had similar profiles of symptoms and mean clinical scores, and Q-CFS/ME was phenotypically similar to the other CFS/ME cases in which the triggering factors were unknown. Patients with endogenous depression had a markedly low prevalence of numbness/tingling and tender lymphadenopathy, and less bodily pain, as indicated by the McGill Pain Questionnaire mean score, as compared with CFS/ME. Normal blood donors had very low prevalence of all symptoms, little fatigue (Chalder), pain (McGill), associated symptoms (Somatic and Psychological Health Report), normal sleep (Pittsburgh Sleep Questionnaire Index) and high SF36 total scores (table 1), as would be expected.

\section{Quantitative PCR}

qPCR was carried out using TaqMan primers/probes specific for 88 human genes that were previously found to be differentially expressed in CFS/ME patients. ${ }^{7}$ This analysis confirmed that most of these genes differed significantly between CFS/ME and the normal group. Of the 88 genes, 84 were found to be upregulated and four were downregulated (HIF1A, IL7R, PAPOLA, $S H P R H)$, which is similar to what we reported previously. ${ }^{7}$ Gene expression in patients with Q-CFS/ME was also found to be markedly different from the normal group, and very similar to that found in patients with CFS/ME. Gene expression in patients with endogenous depression did not differ markedly from that in the normal group, except in the case of five genes (APP, CREBBP, GNAS, $P D C D 2, P D C D 6$ ), where significant upregulation (fold difference $\geq 1.5$ ) was found (table 2 ). 
Table 1 Patient information including age, sex, symptoms and questionnaire results summarising fatigue severity, pain, sleep, general function and associated symptoms for patients with CFS/ME and normal blood donors enrolled in microarray and real-time PCR studies, respectively

\begin{tabular}{|c|c|c|c|c|c|c|}
\hline Variable & $\begin{array}{l}\text { CFS/ME } \\
\text { patients in } \\
\text { previous study } \\
(\mathrm{n}=55)\end{array}$ & $\begin{array}{l}\text { CFS/ME } \\
\text { patients, } \\
\text { previously } \\
\text { untested } \\
(n=56)\end{array}$ & $\begin{array}{l}\text { 0-CFS/ME } \\
\text { patients* } \\
(n=6)\end{array}$ & $\begin{array}{l}\text { All CFS/ME } \\
\text { patients } \\
(\mathrm{n}=117)\end{array}$ & $\begin{array}{l}\text { Patients with } \\
\text { endogenous } \\
\text { depression } \\
(n=14)\end{array}$ & $\begin{array}{l}\text { Normal blood } \\
\text { donors } \\
(\mathrm{n}=29)\end{array}$ \\
\hline Gender (M:F) & 19:36 & $10: 46$ & $6: 0$ & $35: 82$ & $4: 10$ & $14: 15$ \\
\hline Mean age (years) & 41.6 & 40.25 & 41.5 & 41.3 & 41.36 & 44.6 \\
\hline $\begin{array}{l}\text { Mean duration of disease } \\
\text { (years:months) }\end{array}$ & 3.17 & 2.9 & 5.7 & 3.4 & $0: 6$ & NA \\
\hline Headache & 26 & 30 & 1 & 57 & 5 & 1 \\
\hline Sore throat & 27 & 29 & 0 & 56 & 1 & 1 \\
\hline Poor memory/concentration & 30 & 46 & 4 & 80 & 11 & 3 \\
\hline Muscle pain & 37 & 42 & 6 & 85 & 5 & 2 \\
\hline Muscle weakness & 36 & 31 & 5 & 72 & 2 & 1 \\
\hline Fainting/dizziness & 25 & 45 & 5 & 75 & 8 & 1 \\
\hline Numbness/tingling & 24 & 25 & 2 & 51 & 1 & 0 \\
\hline Tender lymphadenopathy & 27 & 22 & 2 & 51 & 0 & 0 \\
\hline \multicolumn{7}{|l|}{ Mean scores } \\
\hline Physical fatigue (Chalder) & 16.13 & 14.36 & 10.83 & 15.15 & 14.00 & 7.69 \\
\hline Mental fatigue (Chalder) & 8.05 & 7.34 & 6.00 & 7.98 & 7.42 & 4.24 \\
\hline McGill Pain Questionnaire & 15.28 & 18.57 & 18.80 & 17.58 & 9.67 & 2.48 \\
\hline Sphere questionnaire & 11.25 & 11.21 & 7.33 & 10.87 & 12.45 & 2.07 \\
\hline SF36 questionnaire & 46.45 & 38.65 & 52.85 & 45.12 & 46.19 & 83.61 \\
\hline $\begin{array}{l}\text { Pittsburgh Sleep } \\
\text { Quality Index }\end{array}$ & 10.22 & 10.00 & 8.17 & 10.01 & 12.25 & 4.28 \\
\hline
\end{tabular}

*These six Q-CFS/ME patients were all part of the 1989 Birmingham Q-CFS/ME outbreak cohort.

CFS/ME, chronic fatigue syndrome/myalgic encephalomyelitis; NA, not applicable; Sphere, Somatic and Psychological Health Report.

\section{Genomic CFS/ME subtypes}

Clustering of $\Delta \mathrm{Ct}$ values for the $88 \mathrm{CFS} / \mathrm{ME}$-associated genes in the $117 \mathrm{CFS} / \mathrm{ME}$ patients identified eight subtypes (designated A-H), consisting of 27, 6, 19, 5, 21, 13, 19 and $4 \mathrm{CFS} / \mathrm{ME}$ patients, respectively. There were three patients whose gene profile did not fit into any of these eight subtype groupings. Mean fold-difference values for each CFS/ME subtype are shown in table 3 and figure 1. Most genes in each subtype were shown to be upregulated (figure 1 and table 3).

The relationship between the subtypes of the present study and those of the previous study which examined only $55 \mathrm{CFS} / \mathrm{ME}$ patients ${ }^{7}$ may be difficult to determine. As these subtypes are derived by using clustering, which finds similar groups on the basis of gene expression values, there is no means to predict the outcome of the clustering. As there was incomplete preservation of the previous CFS/ME patient groupings in the present study, we have designated the subtypes, $\mathrm{A}-\mathrm{H}$, to distinguish them from those of the previous study, which were designated $1-7$.

Analysis of sex ratios for each subtype reveals that subtype D is made up of females only, subtype $\mathrm{H}$ is made up of equal numbers of males and females, and the remaining subtypes are made up predominantly of females.

It is particularly interesting that five of six CFS/ME patients with Q-CFS/ME clustered in the same subtype (subtype A).

The clinical phenotype was distinct between subtypes. Subtype D was the most severe, having the lowest scores for SF36 modules RP, VIT, GH, BP and total score, and the highest frequency of occurrence of muscle pain and sleep problems (see figure 2 for definitions of abbreviations for SF36 modules). Subtype B was the least severe, having the highest scores for SF36 modules RP, GH, MH and total score. Subtype B had a higher median score for the SF36-RP (physical role) than all the others combined (87.5 vs 0), $p=0.04$; Mann-Whitney $U$ test). However, subtype $\mathrm{B}$ had the highest frequency of cognitive dysfunction, muscle weakness and post-exertional malaise. Subtype B showed a higher frequency of cognitive dysfunction than all non-subtype $B$ patients combined $(p=0.03)$ and showed an increased severity and duration of headache compared with all non-subtype $B$ patients combined $(p=0.02)$. Subtype $B$ also had a higher median score for mental fatigue (Chalder scale) than all non-subtype $B$ patients combined, although this did not reach significance $(9.5$ vs 7.0; $p=0.06)$. Subtypes $B$ and $C$ had the best mental health scores, and subtypes A and $\mathrm{F}$ had the worst (figure 2A,B).

Subtype E had a higher median score for SF36-VIT than all the others combined (35.0 vs 15.0; $\mathrm{p}=0.05$; Mann-Whitney U test). Subtype E resulted in the highest frequency of gastrointestinal problems. Patients of subtype F showed a higher frequency of increased severity of numbness/tingling compared with all nonsubtype $F$ patients combined $(p=0.03)$. Patients of subtype $H$ showed an increased frequency of severity of sore throat compared with all non-subtype $H$ patients combined $(p=0.01)$ (figure 2A,B).

As regards possible association of subtype with geographical location, there was evidence to support this, as we found previously. ${ }^{7}$ Predominant subtypes in each geographical location were as follows: Birmingham, subtype A; Bristol, subtype C; 
Table 2 CFS/ME-associated genes and transcription factors in patients with CFS/ME, Q-fever-associated CFS/ME and endogenous depression

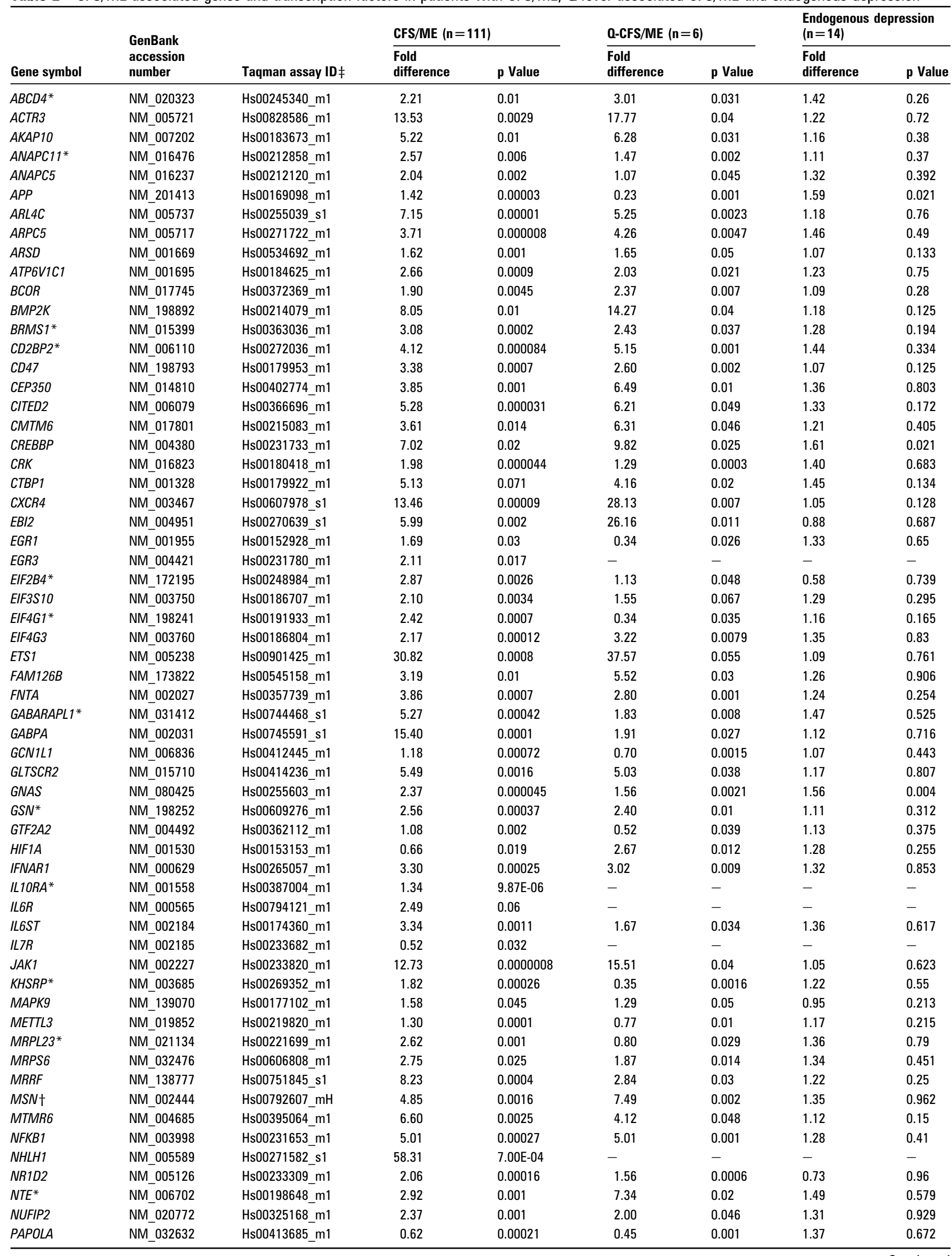


Table 2 Continued

\begin{tabular}{|c|c|c|c|c|c|c|c|c|}
\hline Gene symbol & $\begin{array}{l}\text { GenBank } \\
\text { accession } \\
\text { number }\end{array}$ & Taqman assay ID $\neq$ & \multicolumn{2}{|c|}{ CFS/ME $(n=111)$} & \multicolumn{2}{|c|}{ Q-CFS/ME $(n=6)$} & \multicolumn{2}{|c|}{$\begin{array}{l}\text { Endogenous depression } \\
(\mathrm{n}=14)\end{array}$} \\
\hline PDCD6 & NM_013232 & Hs00737034_m1 & 2.54 & 0.0002 & 2.19 & 0.01 & 1.69 & 0.015 \\
\hline PEX16* & NM_004813 & Hs00191337_m1 & 3.98 & 0.0061 & 3.32 & 0.028 & 0.68 & 0.776 \\
\hline PGM2 & NM_018290 & Hs00217619_m1 & 4.28 & 0.000001 & 3.50 & 0.0014 & 1.07 & 0.308 \\
\hline POLR2G* & NM_002696 & Hs00275738_m1 & 2.71 & 0.001 & 1.00 & 0.039 & 0.77 & 0.916 \\
\hline PPP2R5C & NM_002719 & Hs00604902_m1 & 4.65 & 0.013 & 8.21 & 0.045 & 1.30 & 0.906 \\
\hline PRKAA1 & NM_006251 & Hs01562315_m1 & 4.19 & 0.0002 & 2.18 & 0.001 & 1.29 & 0.56 \\
\hline PRKAR1A & NM_002734 & Hs00267597_m1 & 3.55 & 0.0000004 & 2.31 & 0.0001 & 1.23 & 0.83 \\
\hline PUM2 & NM_015317 & Hs00209677_m1 & 2.73 & 0.00078 & 2.33 & 0.002 & 1.35 & 0.82 \\
\hline$R A P 2 C$ & NM_021183 & Hs00221801_m1 & 6.74 & 0.013 & 4.37 & 0.043 & 1.46 & 0.69 \\
\hline SHPRH & NM_173082 & Hs00542737_m1 & 0.56 & 0.02 & 0.69 & 0.03 & 1.00 & 0.303 \\
\hline SNAP23 & NM_003825 & Hs00187075_m1 & 7.00 & 0.0006 & 3.17 & 0.01 & 1.10 & 0.132 \\
\hline SORL1 & NM_003105 & Hs00268342_m1 & 1.67 & 4.10E-08 & - & - & - & - \\
\hline SOS1 & NM_005633 & Hs00362308_m1 & 1.02 & 0.001 & 1.02 & 0.037 & 1.27 & 0.52 \\
\hline TAF11 & NM_005643 & Hs00194573_m1 & 1.17 & 0.001 & 0.00 & 0.02 & 1.40 & 0.57 \\
\hline TCF3 & NM_003200 & Hs00413032_m1 & 2.40 & 0.03 & 1.63 & 0.068 & 1.29 & 0.86 \\
\hline TDP1 & NM_018319 & Hs00217832_m1 & 3.12 & 0.001 & 3.16 & 0.01 & 1.12 & 0.83 \\
\hline TNFRSF1A & NM_001065 & Hs00533560_m1 & 12.37 & 0.004 & 18.52 & 0.03 & 0.86 & 0.279 \\
\hline UBTF & NM_014233 & Hs00610729_g1 & 6.38 & 0.002 & 2.40 & 0.011 & 1.09 & 0.297 \\
\hline USP38 & NM_032557 & Hs00261419_m1 & 3.35 & 0.01 & 4.98 & 0.078 & 1.43 & 0.367 \\
\hline WAPAL & NM_015045 & Hs00386162_m1 & 3.94 & 0.003 & 3.69 & 0.026 & 1.17 & 0.44 \\
\hline
\end{tabular}

*Genes found in pilot study. ${ }^{13}$

†Genes found in study using differential display/PCR. ${ }^{7}$

$¥$ Taqman assays were those pre-designed by Applied Biosystems, Warrington, UK.

$\mathrm{CFS} / \mathrm{ME}$, chronic fatigue syndrome/myalgic encephalomyelitis.

Leicester, subtype C; London, subtype C, then subtype G; New York, subtype E, then subtypes G, A, C and F; Dorset, subtypes A, F, B. Subtype A was prominent in New York, Birmingham and Dorset; subtype B was prominent in Dorset; subtype C was prominent in Bristol, London and New York; subtype D was prominent in Bristol and London; subtype E was prominent in New York; subtype F was prominent in Dorset and New York; subtype $\mathrm{G}$ was prominent in New York; subtype $\mathrm{H}$ was prominent in Dorset (figure 2C).

\section{Microbial infections}

The presence and titre of specific antibodies ( $\operatorname{IgM}$ and $\operatorname{IgG}$ ) to four treatable microbial infections that are well recognised as triggers of CFS/ME were also determined in serum samples; these were EBV, enterovirus, parvovirus B19 and C burnetii. The seroprevalence (proportion of subjects who were positive for specific $\operatorname{IgG}$ ) of each of these infections was typical of the general population: EBV (based on VCA IgG), 88\%; enterovirus, 49\%; parvovirus B19 (based on viral protein 2 IgG), 74\%; C burnetii (based on phase I or II IgG), $10 \%$. Of the 11 patients who had $C$ burnetii IgG, five were patients whose CFS/ME disease had been triggered by laboratory-documented $\mathrm{O}$ fever.

CFS/ME patients with acute infection with one or more of these agents (IgM or acute phase IgG) were also detected: EBV (based on VCA $\operatorname{IgM})(\mathrm{n}=3)$, enterovirus $(\mathrm{n}=6)$, parvovirus B19 $(\mathrm{n}=1)$, $C$ burnetii (based on phase II IgG) $(n=12)$. Of the 12 patients who were positive for $C$ burnetii phase II IgG, five had Q-CFS/ME. There were no acute infections detected in the normal group.
Regarding EBV serology, there were also associations between CFS/ME subtype and both EBV VCA IgM titre $(p=0.0038)$ and EBV EBNA IgG titre $(p=0.0011)$ (figure 2D). Using the EBV markers VCA IgM, VCA IgG, early antigen IgG and EBNA IgG, we determined the EBV serostatus of infection for each subject (ie, seronegative, primary infection/re-activation, late phase of infection). Among 111 of these CFS/ME patients, there were 11 seronegative, 61 primary/re-activation and 39 late phase of infection, as compared with the normal group, in which there was one seronegative, eight primary/re-activation, and 19 late phase of infection $\left(\chi^{2}=9.91\right.$, degrees of freedom $=2, p=0.007$ ) (figure $2 E$ ).

The distribution of CFS/ME patients by EBV serostatus category (seronegative, primary/re-activation and late phase of infection) across the eight CFS/ME genomic subtypes is shown in figure $2 \mathrm{E}$. In the normal controls, the predominant category of EBV serostatus was late phase of infection, whereas in the CFS/ME subtypes, the predominant category of EBV serostatus was primary/re-activation, which was seen in subtypes $A, B, C$, $\mathrm{D}, \mathrm{F}$ and $\mathrm{H}$. Subtype $\mathrm{G}$ had equal numbers of primary/re-activation and late phase, and subtype $\mathrm{E}$ had a predominance of late phase subjects, but also had five seronegative subjects. This distribution was found to be almost statistically significant $\left(\chi^{2}=25.9\right.$, degrees of freedom $\left.=16, p=0.055\right)$.

\section{EBV-associated genes in each CFS/ME subtype}

Within the CFS/ME-associated gene signature of 88 human genes, there were 12 that have recognised associations with EBV infection; these associations have been summarised previously. ${ }^{7}$ 
Table 3 Fold-difference values for 88 genes in each of eight subtypes $(A-H)$ in 114 subtyped patients with chronic fatigue syndrome/myalgic encephalomyelitis (CFS/ME). Genes without values for the subtypes are those for which there was missing data for one or more subtypes. Bold type indicates genes targeted by existing drugs and those CFS/ME subtypes in which fold-difference values of 1.5 were found

\begin{tabular}{|c|c|c|c|c|c|c|c|c|c|}
\hline \multirow{2}{*}{$\begin{array}{l}\text { Gene } \\
\text { symbol }\end{array}$} & \multirow{2}{*}{$\begin{array}{l}\text { Genbank } \\
\text { accession }\end{array}$} & \multicolumn{8}{|c|}{ CFS/ME subtype } \\
\hline & & $\bar{A}$ & B & C & D & $E$ & $\mathbf{F}$ & G & H \\
\hline$A B C D 4$ & NM_020323 & 1.40 & 0.60 & 0.81 & 4.40 & 10.61 & 7.02 & 1.32 & 0.93 \\
\hline ACTR3 & NM_005721 & 10.17 & 8.03 & 4.34 & 25.73 & 13.95 & 6.75 & 27.57 & 5.35 \\
\hline ANAPC11 & NM_016476 & 2.53 & 2.66 & 4.34 & 3.74 & 1.59 & 4.16 & 2.27 & \\
\hline ANAPC5 & NM_016237 & 1.13 & 1.26 & 4.49 & 1.92 & 0.78 & 0.00 & 2.96 & 2.95 \\
\hline$A P P$ & NM_201413 & 0.63 & 1.73 & 1.42 & 0.56 & 4.64 & 0.84 & 0.84 & 2.58 \\
\hline$A R S D$ & NM_001669 & 0.64 & 1.17 & 1.51 & 4.60 & 2.62 & 0.26 & 0.12 & 2.19 \\
\hline ATP6V1C1 & NM_001695 & 1.99 & 3.80 & 3.38 & 2.43 & 2.06 & 0.72 & 3.63 & 4.55 \\
\hline$B C O R$ & NM_017745 & 1.42 & 2.22 & 1.78 & 2.30 & 6.52 & 1.13 & 2.65 & 2.57 \\
\hline BMP2K & NM_1988892 & 8.96 & 4.83 & 2.62 & 16.42 & 7.76 & 3.78 & 11.57 & 2.06 \\
\hline BRMS1 & NM_015399 & 1.31 & 4.38 & 2.44 & 1.53 & 4.62 & 7.06 & 2.57 & 3.83 \\
\hline СMTM6 & NM_017801 & 3.10 & 3.70 & 0.69 & 10.04 & 7.81 & 4.77 & 1.71 & 0.73 \\
\hline CREBBP & NM_004380 & 7.11 & 1.62 & 1.09 & 13.34 & 5.46 & 2.61 & 8.99 & 3.62 \\
\hline CRK & NM_016823 & 1.83 & 5.57 & 1.26 & 2.82 & 4.89 & 1.03 & 2.25 & 2.02 \\
\hline CTBP1 & NM_001328 & 4.95 & 4.98 & 1.05 & 8.62 & 15.44 & 3.43 & 2.42 & 2.73 \\
\hline CXCR4 & NM_003467 & 13.47 & 2.18 & 2.03 & 28.10 & 17.57 & 1.48 & 10.29 & 3.57 \\
\hline$E B / 2$ & NM_004951 & 5.67 & 1.41 & 2.31 & 14.93 & 5.99 & 0.76 & 0.42 & 4.47 \\
\hline EGR1 & NM_001955 & 0.49 & 2.85 & 2.42 & 0.30 & 0.27 & 1.00 & 1.98 & 2.96 \\
\hline EGR3 & NM_004421 & 0.95 & 1.33 & 0.46 & & 5.36 & & 1.39 & 0.98 \\
\hline EIF2B4 & NM_172195 & 1.44 & 0.52 & 1.33 & 6.00 & 3.48 & 0.15 & 1.69 & 2.08 \\
\hline EIF3S10 & NM_003750 & 1.43 & 4.42 & 2.10 & 1.72 & 1.48 & 1.25 & 2.83 & 6.16 \\
\hline EIF4G1 & NM_198241 & 1.13 & 3.47 & 3.52 & 0.99 & 1.39 & 2.30 & 4.53 & 14.27 \\
\hline EIF4G3 & NM_003760 & 2.40 & 0.79 & 0.77 & 5.46 & 27.42 & 1.09 & 3.62 & 1.55 \\
\hline GSN & NM_198252 & 1.73 & 1.27 & 1.69 & 3.82 & 2.36 & 1.64 & 3.51 & 5.81 \\
\hline GTF2A2 & NM_004492 & 0.71 & 0.53 & 1.21 & 0.53 & 2.19 & 0.48 & 1.65 & 0.87 \\
\hline HIF1A & NM_001530 & 2.04 & 0.87 & 0.82 & 5.14 & 4.22 & 1.66 & 4.65 & 1.35 \\
\hline IFNAR1 & NM_000629 & 1.86 & 0.17 & 0.79 & 3.55 & 5.53 & 1.41 & 7.17 & 0.91 \\
\hline IL10RA & NM_001558 & 1.12 & 2.68 & 0.74 & & & 2.31 & 1.01 & 1.76 \\
\hline IL6R & NM_-000565 & 2.19 & & 2.47 & & & 2.78 & 2.67 & \\
\hline IL6ST & NM_002184 & 2.61 & 1.49 & 4.81 & 3.14 & 3.07 & 2.67 & 3.76 & \\
\hline$I L 7 R$ & NM_002185 & 1.43 & 0.91 & 1.66 & & & 1.46 & 2.06 & 1.33 \\
\hline JAK1 & NM_002227 & 9.72 & 7.84 & 3.29 & 28.88 & 9.80 & 11.17 & 18.75 & 6.19 \\
\hline KHSRP & NM_003685 & 0.42 & 1.03 & 0.91 & 0.61 & 1.15 & 0.75 & 1.07 & 1.62 \\
\hline МАРК & NM_139070 & 1.16 & & 1.62 & 1.68 & 1.83 & 0.00 & 2.51 & \\
\hline METTL3 & NM_019852 & 0.81 & 1.38 & 0.64 & 1.72 & 2.92 & 1.08 & 1.81 & 0.68 \\
\hline MRPL23 & NM_021134 & 2.34 & 0.97 & 1.23 & 4.15 & 4.20 & 1.56 & 2.56 & 2.06 \\
\hline MRPS6 & NM_032476 & 2.03 & 0.92 & 2.93 & 3.05 & 7.75 & 1.77 & 1.95 & 2.00 \\
\hline MRRF & NM_138777 & 10.11 & 13.30 & 3.96 & 2.03 & 9.28 & 1.33 & 9.70 & 7.34 \\
\hline MSN & NM_002444 & 3.20 & 1.66 & 1.81 & 9.47 & 7.86 & 3.13 & 8.58 & 2.12 \\
\hline MTMR6 & NM_004685 & 3.71 & 11.73 & 2.61 & 7.73 & 7.33 & 2.67 & 14.97 & 2.58 \\
\hline$N F K B 1$ & NM_003998 & 3.74 & 0.91 & 0.65 & 8.83 & 6.51 & 4.10 & 7.30 & 1.55 \\
\hline NHLH1 & NM_005589 & 26.32 & 37.92 & 49.09 & & & 66.39 & 51.25 & 126.29 \\
\hline NR1D2 & NM_005126 & 1.40 & & 2.31 & 4.57 & 3.69 & 1.27 & 2.50 & 2.29 \\
\hline NTE & NM_006702 & 1.75 & 0.31 & 0.89 & 3.92 & 4.37 & 1.30 & 3.87 & 1.43 \\
\hline NUFIP2 & NM_020772 & 1.55 & 1.90 & 1.81 & 2.83 & 2.10 & 1.84 & 3.50 & 2.31 \\
\hline
\end{tabular}


Table 3 Continued

\begin{tabular}{|c|c|c|c|c|c|c|c|c|c|}
\hline \multirow{2}{*}{$\begin{array}{l}\text { Gene } \\
\text { symbol } \\
\end{array}$} & \multirow{2}{*}{$\begin{array}{l}\text { Genbank } \\
\text { accession }\end{array}$} & \multicolumn{8}{|c|}{ CFS/ME subtype } \\
\hline & & $\bar{A}$ & B & C & D & $\mathbf{E}$ & $\mathbf{F}$ & G & H \\
\hline PAPOLA & NM 032632 & 0.47 & 0.52 & 0.29 & 0.79 & 4.73 & 0.58 & 1.25 & 0.32 \\
\hline PDCD2 & NM_002598 & 3.83 & 3.44 & 2.94 & 5.00 & 5.74 & 5.88 & 5.36 & 7.37 \\
\hline PDCD6 & NM_013232 & 1.96 & 2.72 & 2.53 & 2.16 & 4.79 & 2.69 & 2.85 & 2.16 \\
\hline PEX16 & NM_004813 & 2.10 & 16.10 & 2.04 & 8.88 & 5.92 & 0.00 & 2.90 & 2.80 \\
\hline PGM2 & NM_018290 & 3.23 & 3.62 & 2.16 & 5.99 & 5.72 & 4.89 & 6.13 & 3.36 \\
\hline PIK3R1 & NM_181523 & 2.06 & 4.55 & 0.58 & 7.17 & 7.31 & 0.95 & 5.48 & 0.82 \\
\hline POLR2G & NM_002696 & 1.09 & 5.58 & 2.06 & 1.91 & 6.01 & 2.60 & 3.82 & 2.91 \\
\hline PPP2R5C & NM_002719 & 2.78 & 2.62 & 1.28 & 9.50 & 6.14 & 1.63 & 7.87 & 0.84 \\
\hline PRKAA1 & NM_006251 & 2.14 & 4.10 & 3.42 & 3.53 & 4.17 & 6.87 & 7.13 & 3.11 \\
\hline PRKAR1A & NM_002734 & 2.05 & 1.85 & 2.56 & 4.14 & 3.66 & 2.41 & 6.35 & 5.41 \\
\hline PUM2 & NM_015317 & 2.81 & 1.69 & 0.87 & 2.85 & 5.04 & 1.49 & 3.84 & 2.22 \\
\hline$R A P 2 C$ & NM_021183 & 2.69 & & 2.56 & 4.75 & 5.35 & 25.28 & 10.61 & 1.95 \\
\hline SHPRH & NM_173082 & 0.82 & & 1.07 & 0.21 & 7.17 & 0.00 & 0.64 & \\
\hline SNAP23 & NM_003825 & 3.46 & 0.45 & 1.89 & 12.62 & 13.33 & 4.15 & 10.19 & 1.43 \\
\hline SORL1 & NM_003105 & 1.40 & 1.91 & 1.60 & & & 2.01 & 1.52 & 2.47 \\
\hline SOS1 & NM_005633 & 0.70 & & 0.81 & 1.69 & 1.09 & 0.29 & 1.61 & 0.90 \\
\hline TAF11 & NM_005643 & 0.56 & & 1.35 & 1.05 & 2.13 & 0.00 & 0.21 & 1.23 \\
\hline TCF3 & NM_003200 & 2.00 & 0.94 & 1.08 & 2.83 & 3.96 & 3.54 & 2.52 & 2.65 \\
\hline$T D P 1$ & NM_018319 & 1.60 & 5.50 & 1.38 & 4.80 & 11.55 & 0.96 & 4.24 & \\
\hline TNFRSF1A & NM_001065 & 11.96 & 4.07 & 1.36 & 18.01 & 13.25 & 3.30 & 17.81 & 2.06 \\
\hline UBTF & NM_014233 & 2.88 & 3.59 & 1.82 & 6.03 & 6.46 & 4.81 & 10.91 & 6.44 \\
\hline USP38 & NM_032557 & 2.66 & & 0.71 & 7.40 & 4.27 & 7.18 & 2.94 & 1.02 \\
\hline WAPAL & NM_015045 & 2.97 & & 5.13 & 3.63 & 2.78 & 1.24 & 6.04 & 2.97 \\
\hline WDR26 & NM_025160 & 0.84 & 0.09 & 0.63 & 2.18 & 1.53 & 0.80 & 2.74 & 1.23 \\
\hline
\end{tabular}

The fold-difference values for each of these 12 genes in each CFS/ME subtype/normal were analysed for significant associations using ANOVA. With all 12 genes, there was a trend which did not reach significance $(\mathrm{df}=89, \mathrm{p}=0.119)$. However, when GABPA and $E G R 1$ were removed from the analysis, the remaining 10 genes showed a striking association with subtype (ANOVA, df $=73$, $\mathrm{p}=0.0001$ ) (figure $2 \mathrm{~F}$ ).

\section{DISCUSSION}

We have previously reported the differential expression of 88 human genes in CFS/ME and evidence of clinically relevant subtypes. ${ }^{7} 8$ In the present study, we have confirmed this differential expression in 62 additional and previously untested CFS/ME patients. Combining the previous cohort and the new cohort, we have found evidence of eight genomic CFS/ME subtypes with marked differences in global functioning, clinical symptoms, levels of severity and geographical distribution. The function of these genes and their networks has been published previously. ${ }^{7}$

We have addressed the question of the specificity of these 88 genes to CFS/ME, by testing drug-free patients with endogenous depression. The fact that only five of these genes were

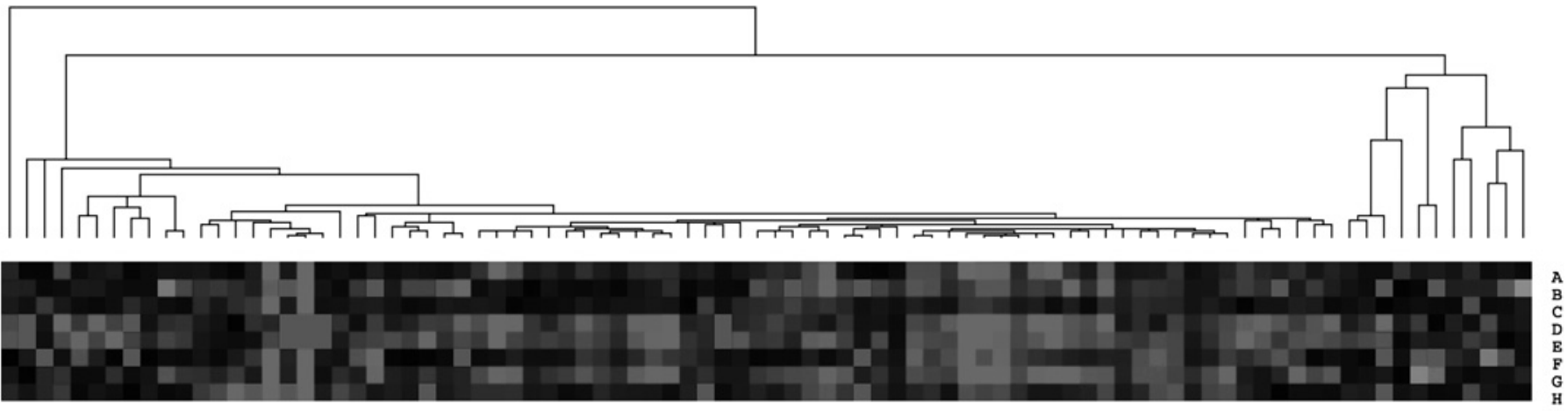

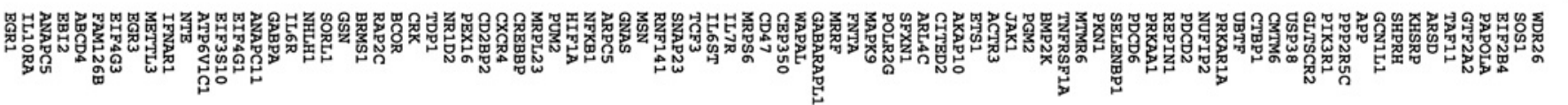

Figure 1 Absolute fold-difference values (mean relative quantity (RO) in patients with chronic fatigue syndrome/myalgic encephalomyelitis (CFS/ME)/ mean $\mathrm{RO}$ in normal controls) for each of $88 \mathrm{CFS} / \mathrm{ME}$-associated genes in eight CFS/ME subtypes (A-H). 

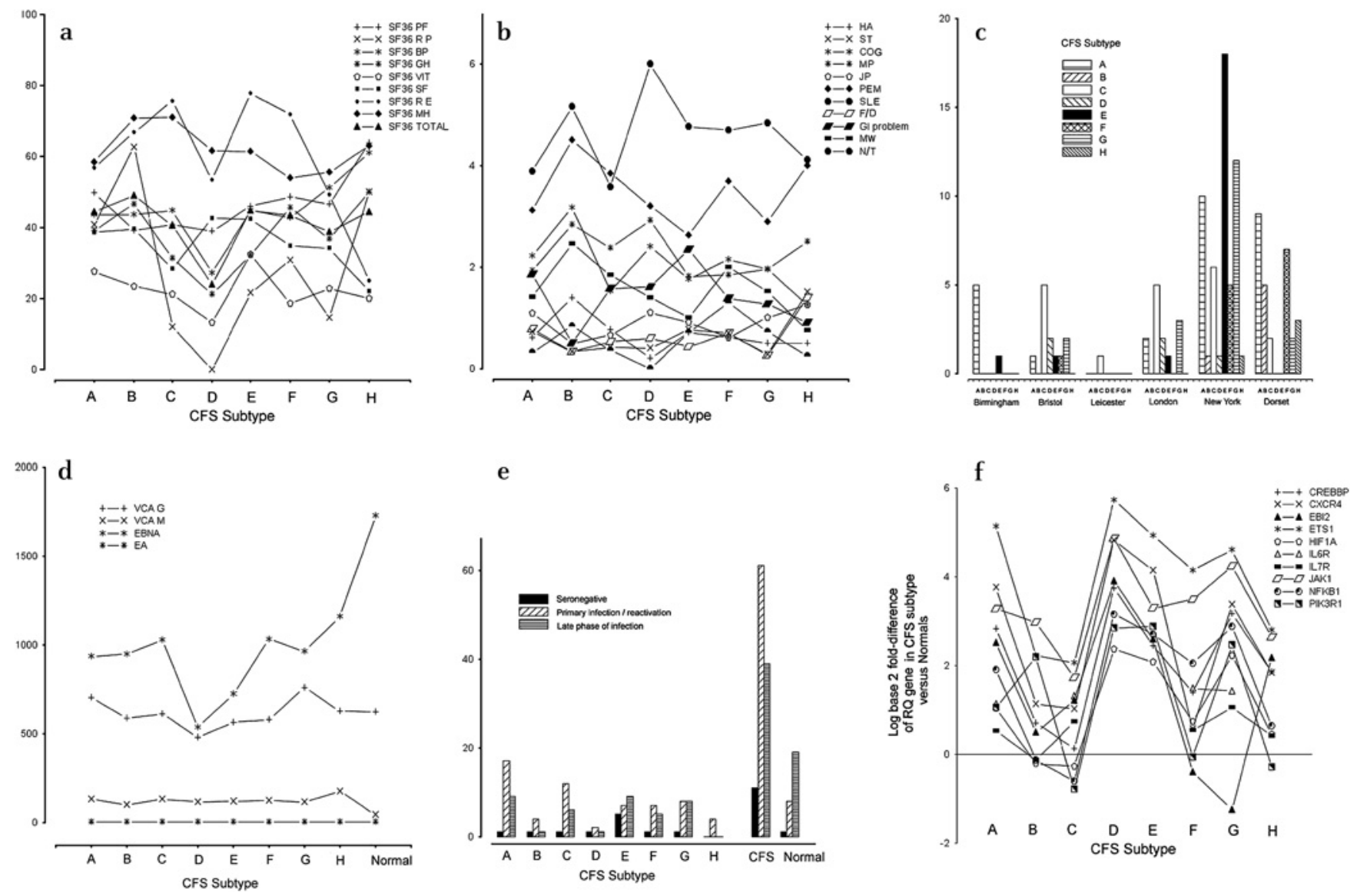

Figure 2 (a) Medical Outcomes Survey Short Form-36 (SF36) domain and total scores for each chronic fatigue syndrome/myalgic encephalomyelitis (CFS/ME) subtype: physical function, physical role (RP), bodily pain (BP), general health (GH), vitality (VIT), social functioning (SF), emotional role (RE), mental health (MH) and total score (Total). (b) Scores indicating occurrence and severity of 11 clinical symptoms for each CFS/ME subtype: headache (HA), sore throat (ST), swollen glands (GLA), cognitive defect (COG), muscle pain (MP), joint pain (JP), muscle weakness (MW), post-exertional malaise (PEM), sleep problems (SLE), fainting/dizziness (F/D), gastrointestinal complaints (GI), numbness/tingling (N/T), spatial span (SSP), verbal recognition memory (VRM). (c) Histogram showing the numbers of CFS/ME patients of each subtype occurring in each of the six geographical locations. (d) Epstein-Barr virus (EBV) antibody titres (viral capsid antigen (VCA) IgM, VCA IgG, early antigen (EA) IgG, Epstein-Barr nuclear antigen (EBNA) $\mathrm{IgG}$ ) in each CFS/ME subtype and the normal comparison group. (e) Distribution of categories of EBV serostatus (seronegative, primary/re-activation, late phase of infection) in the CFS/ME subtypes, A-H, in CFS/ME (all subtypes combined) and in normal controls. (f) Log (base 2) of fold-difference values of 10 human genes known to be important in EBV infection, in eight CFS subtypes (A-H).

abnormally expressed in these patients, as compared with normal controls, supports the view that CFS/ME and endogenous depression are biologically distinct, and that the psychological features of CFS/ME are in fact secondary to the pathogenesis.

It is particularly interesting that five of six CFS/ME patients with $\mathrm{Q}-\mathrm{CFS} / \mathrm{ME}$ clustered in the same subtype (subtype A). As these patients had had CFS/ME for several years, this finding suggests that they have a common underlying theme, which may be stable for a long time after the onset of disease. In view of this, and as various genes within this human gene signature are closely linked with EBV infection (NFKB1, EGR1, ETS1, GABPA, CREBBP, CXCR4, EBI2, HIF1A, JAK1, IL6R, IL7R, $P I K 3 R 1)$ and enterovirus infection (EIF4G1), we tested the serum samples for markers for four treatable microbial infections that are well recognised to trigger CFS/ME (EBV, enterovirus, parvovirus $\mathrm{B} 19$ and $C$ burnetii (the agent of $\mathrm{Q}$ fever)) with the hypothesis that these genomic CFS/ME subtypes may represent host responses to particular infectious agents.

One patient with subtype E had acute parvovirus B19 at the time of sampling. This patient's symptoms were typical of CFS/ME, but this is not unexpected as parvovirus B19 is a recognised trigger for
$\mathrm{CFS} / \mathrm{ME}^{13}$ The importance of testing for these infections is illustrated here, as we have shown previously that B19-CFS/ME is highly responsive to treatment with intravenous immunoglobulin. ${ }^{14}$

Six patients had acute enterovirus infections (of undetermined serotype) at the time of sampling, but there was no subtype relationship, as two patients were found to have each of subtypes A, E and G. Enteroviruses have long been recognised to trigger CFS/ME, ${ }^{15}$ and they have been detected in the stool ${ }^{16}$ and stomach epithelium ${ }^{17}$ in CFS/ME patients. Detection in the stomach has been shown to be associated with gastrointestinal symptoms in CFS/ME patients. ${ }^{17}$ However, in the present study, patients of subtypes A, E and G did not show gastrointestinal symptoms more often than the other patients.

Twelve CFS/ME patients and one normal subject had IgG to $C$ burnetii phase II antigen, suggesting possible acute infection. Five of these CFS/ME patients were among those with Q-CFS/ME. The patients in whom these antibodies were detected had subtypes A, B, D, E and G. Therefore, apart from the patients with Q-CFS/ME (whose CFS/ME disease onset was associated with laboratory-documented acute $\mathrm{Q}$ fever), there were no subtypespecific relationships with $C$ burnetii antibodies. 


\section{Take-home messages}

- Expression of 88 human genes was confirmed as being significantly different between patients with chronic fatigue syndrome/myalgic encephalomyelitis (CFS/ME) and normal controls.

- Gene expression in patients with endogenous depression was similar to that in normal controls.

- CFS/ME patients can be grouped into genomic subtypes which have different clinical phenotypes.

- There was evidence of subtype-specific relationships for Epstein-Barr virus and enterovirus, the two most common triggers for CFS/ME.

The subtype associations with EBV and EBV-linked genes are interesting, suggesting differences in the role of EBV and consequent host responses in the different subtypes. The finding of a noticeably large proportion of CFS/ME patients who were EBV seronegative $(10 \%)$, compared with $4 \%$ in the normal group, was quite surprising given the strong link between EBV and CFS/ME. The fact that five of these 11 seronegative cases were subtype $\mathrm{E}$ is interesting, but remains unexplained at present.

It has been recognised for some time that subtypes of CFS/ME exist, and it has been thought that these subtypes may, at least in part, reflect particular aetiological factors. ${ }^{18}$ A symptom-based approach has had some success in identifying musculoskeletal, inflammatory and neurological subtypes ${ }^{19}$; however, these groups had only minor differences in overall functional severity in contrast with those of the present study.

It is intriguing that it is possible to identify CFS/ME subtypes on the basis of expression values for these 88 genes, and even more so that these subtypes have distinct clinical phenotypes, with marked differences in the occurrence of particular symptoms and their severity. However, what precise sequence of events is involved in the genesis of the gene signatures in each subtype remains to be elucidated. Further work is required to validate and develop these findings.

Acknowledgements This work was supported by grants from the Chronic Fatigue Syndrome Research Foundation (CFSRF), UK (salaries of JG and DC), Sir Joseph Hotung (salary of JRK), and a Wellcome Trust Vacation Scholarship (awarded to LZ). We thank Dr Frank Boulton, Ms Julie Williams, Mr Peter Rogers, Ms Diana Carr and the NBS teams of East Dorset for their help in enrolment and sampling of normal blood donors, Beverley Burke, Deepika Devanur, Joanne Hunt and Robert Petty for help with sample processing and omission of non-concordant Q-PCR data for previously published data, and all the patients with CFS/ME and blood donors for their participation.

Funding Wellcome Trust; CFS Research Foundation.

Competing interests None.

Ethics approval This study was conducted with the approval of the Wandsworth Research Ethics Committee, St George's Hospital.

Patient consent Obtained.

Provenance and peer review Not commissioned; externally peer reviewed.

\section{REFERENCES}

1. Fukuda K, Straus SE, Hickie I, et al. The chronic fatigue syndrome: a comprehensive approach to its definition and study. International Chronic Fatigue Syndrome Study Group. Ann Intern Med 1994;121:953-9.

2. A report of the CFS/ME Working Group. Department of Health January 2002. http://www.dh.gov.uk.

3. Papanicolaou DA, Amsterdam JD, Levine $S$, et al. Neuroendocrine aspects of chronic fatigue syndrome. Neuroimmunomodulation 2004;11:65-74.

4. Komaroff AL, Buchwald D. Chronic fatigue syndrome: an update. Annu Rev Med 1998:49:1-13

5. Devanur LD, Kerr JR. Chronic fatigue syndrome. J Clin Virol 2006;37:139-50.

6. Kaushik N, Fear D, Richards SC, et al. Gene expression in peripheral blood mononuclear cells from patients with chronic fatigue syndrome. J Clin Patho 2005; 58:826-32.

7. Kerr JR, Petty R, Burke B, et al. Differentially expressed genes in chronic fatigue syndrome/myalgic encephalomyelitis (CFS/ME) patients reveal seven subtypes with distinct clinical phenotypes. J Infect Dis 2008;197:1171-84.

8. Kerr JR, Burke B, Petty R, et al. Seven genomic subtypes of chronic fatigue syndrome/myalgic encephalomyelitis: a detailed analysis of gene networks and clinical phenotypes. J Clin Pathol 2008;61:730-9.

9. Reeves WC, Lloyd A, Vernon SD, et al. Identification of ambiguities in the 1994 chronic fatigue syndrome research case definition and recommendations for resolution. BMC Health Serv Res 2003;3:25.

10. Chalder T, Berelowitz G, Pawlikowska T, et al. Development of a fatigue scale. J Psychosom Res 1993;37:147-53.

11. Sturn A, Quackenbush J, Trajanoski Z. Genesis: cluster analysis of microarray data Bioinformatics 2002;18:207-8.

12. Eisen MB, Spellman PT, Brown PO, et al. Cluster analysis and display of genomewide expression patterns. Proc Natl Acad Sci U S A 1998;95:14863-8.

13. Kerr JR, Bracewell J, Laing I, et al. Chronic fatigue syndrome (CFS) and arthralgia following parvovirus B19 infection. J Rheumatol 2002;29:595-602.

14. Kerr JR, Cunniffe VS, Kelleher $P$, et al. Successful intravenous immunoglobulin therapy in 3 cases of parvovirus B19-associated chronic fatigue syndrome. Clin Infect Dis 2003;36:e100-6.

15. Kerr JR. Enterovirus infection of the stomach in chronic fatigue syndrome/myalgic encephalomyelitis. J Clin Pathol 2008;61:1-2.

16. Yousef GE, Bell EJ, Mann GF, et al. Chronic enterovirus infection in patients with postviral fatigue syndrome. Lancet 1988;1:146-50.

17. Chia JK, Chia AY. Chronic fatigue syndrome is associated with chronic enterovirus infection of the stomach. J Clin Pathol 2008;61:43-8.

18. Jason LA, Corradi K, Torres-Harding $S$, et al. Chronic fatigue syndrome: the need for subtypes. Neuropsychol Rev 2005;15:29-58.

19. Janal MN, Ciccone DS, Natelson BH. Sub-typing CFS patients on the basis of 'minor' symptoms. Biol Psychol 2006;73:124-31. 